\title{
OSSERVAZIONI COMPARATIVE SULLA SCRITTURA EPISTOLARE DI BELLINI E DI VERDI
}

\author{
Nota del m.e. ILARIA BONOMI (*)
}

(Adunanza del 4 aprile 2019)

SunTO. - In questo contributo si esamina la scrittura epistolare di Vincenzo Bellini e di Giuseppe Verdi relativamente ad alcuni aspetti linguistici e con particolare riferimento alla corrispondenza con i librettisti delle loro opere.

$* * *$

ABSTRACT. - In this note the author analyses the language of Bellini's and Verdi's letters, with a special reference to the poets of their operas.

Secondo Verdi, le lettere dei musicisti non dovrebbero essere studiate e pubblicate: lo scrisse, con il suo solito stile diretto e veemente, a proposito della pubblicazione di lettere di Bellini in una lettera famosa all'amico Opprandino Arrivabene del 18 ottobre 1880:

Ma che necessità vi è d'andar a tirar fuori delle lettere d'un Maestro di musica? Lettere che sono sempre scritte di fretta, senza cura, senza importanza, perché il maestro sa che non deve sostenere una reputazione di letterato. Non basta che lo fischino per le note? Non signore! Anche le lettere! Ah! È una gran seccatura la celebrità! Questi poveri piccoli-grandi uomini celebri pagano ben cara la popolarità! Mai per essi un'ora di pace né in vita né in morte! ${ }^{1}$

(*) Università degli Studi di Milano, Italy. E-mail: ilaria.bonomi@unimi.it

1 Turba 2018, p. 160. 
Ma noi sappiamo che i motivi per pubblicare e studiare le lettere dei grandi compositori sono tanti e forti. Oltre agli ovvi motivi legati ai contenuti e alle molte informazioni che emergono dalle lettere sugli aspetti compositivi e sui rapporti di collaborazione (in particolare con $\mathrm{i}$ loro librettisti), e oltre all'ovvio interesse di ciò che emerge della loro personalità, sotto il profilo linguistico offrono esempi importanti di scrittura epistolare di scriventi di media cultura; la provenienza regionale costituisce, per l'epoca, un motivo di interesse in più. Così, possiamo dire che le ragioni per studiare le lettere dei grandi compositori sono di ordine musicale, linguistico, e linguistico-musicale, cioè relativo al rapporto tra parole e musica.

All'epistolario di Bellini mi sono accostata in occasione della presentazione, in questa sede, del suo Carteggio, edito nel 2017 dalla Fondazione Bellini per le eccellenti cure di Graziella Seminara; ${ }^{2}$ quello di Verdi, pane quotidiano per chi si occupi della sua opera da ogni punto di vista, compreso quello linguistico, è stato oggetto di diversi studi, ${ }^{3}$ a cui si aggiunge un mio recente contributo, ${ }^{4}$ relativo alle modalità di allocuzione. Dello straordinario interesse delle lettere verdiane, poi, continuiamo ad avere conferme da più parti: ricordo tra l'altro il recente bel libro di Alberto Mattioli Meno grigi più Verdi. ${ }^{5}$ Mi piace infine sottolineare che ad analisi comparative di epistolari di grandi compositori sollecitava anni fa Pier Vincenzo Mengaldo nel suo studio sulla lingua delle lettere verdiane. ${ }^{6}$

2 Bellini 2017: la presentazione del volume si è tenuta all'Istituto Lombardo il 19 ottobre 2017 con interventi del Presidente del Comitato scientifico del Centro di Documentazione per gli Studi Belliniani-Fondazione Bellini Fabrizio Della Seta, di Pierluigi Ledda, Gabriele Dotto, Emilio Sala, Graziella Seminara e di chi scrive. Sulla lingua delle lettere di Bellini, oltre all'Introduzione della Seminara, si veda soprattutto Nicolodi 2004, la cui analisi si basava sull'edizione delle lettere a cura di Luisa Cambi, del 1943: sarebbe opportuna ora una nuova analisi basata sulla più recente, completa e affidabile edizione della Fondazione Bellini.

3 Macinante 1995, Serianni 2002 (ma già 1996), Mengaldo 2003; importante anche Antonelli 2004, che raccoglie e studia dal punto di vista linguistico un corpus di lettere scritte nell'800 da vari autori, tra i quali figura anche Verdi.

4 Bonomi 2020.

5 Mattioli 2018, recensito molto positivamente da Quirino Principe sul Sole Domenicale del 24-3-19.

6 Mengaldo 2003 p. 45. 
Un breve cenno alle edizioni di riferimento: se per Bellini disponiamo ora della pregevole edizione curata da Graziella Seminara, per Verdi abbiamo l'edizione antologica curata per Einaudi da Eduardo Rescigno $^{7}$ e naturalmente gli ormai numerosi volumi dei carteggi con singoli corrispondenti dell'Edizione Nazionale, promossa dall'Istituto Nazionale di Studi Verdiani, edizioni filologicamente impeccabili, ispirate a criteri conservativi.

La ricerca comparativa che qui presento, originata dai due contributi sopra citati per Bellini e Verdi, si basa su un'indagine svolta sulle lettere ad alcuni destinatari, con particolare attenzione per i librettisti: per Bellini Felice Romani e Carlo Pepoli, oltre a Francesco Florimo, interlocutore privilegiato del compositore e primo editore delle sue lettere, il quale come si sa operò sulle lettere di Bellini tagli e pesanti interventi di rimaneggiamento, e ne modificò anche la lingua, con l'intento di affinarla; per Verdi ho seguito in particolare le lettere ai sette librettisti con cui collaborò, e alcuni dei più importanti tra gli amici, Vincenzo Luccardi, Opprandino Arrivabene, e il suocero Antonio Barezzi. ${ }^{8}$

Premessa basilare per l'indagine linguistica è relativa alla competenza linguistica dei due scriventi, in relazione al loro livello culturale (da cui naturalmente resta escluso l'aspetto musicale).

Di Bellini non si sa molto:' scarse e inaffidabili informazioni sull'infanzia e l'adolescenza inducono a collocare una formazione letteraria, pur molto scarsa, solo negli anni degli studi al Collegio Musicale di San Sebastiano di Napoli, dove si formò. Nel complesso la sua cultura letteraria fu abbastanza modesta, rispetto a quella musicale, ma l'epistolario mostra una crescita a partire dal soggiorno milanese, in ulteriore sviluppo durante i soggiorni a Londra e a Parigi, soprattutto in relazione alla lettura di testi letterari e teatrali in vista della scelta dei soggetti delle opere. Né sappiamo molto di come si formò la sua educazione alla lingua italiana e delle sue preoccupazioni in tal senso, considerando che

7 Verdi 2012.

8 Mi sono basata su Verdi 2012, e sui seguenti carteggi delle Edizioni Nazionali: Cammarano 2001, Somma 2003, Luccardi 2008, Boito 2014.

9 Oltre alle informazioni contenute nell'Introduzione di Graziella Seminara ai Carteggi, si veda soprattutto Pastura 1959; cfr. anche Nicolodi 2004. 
la sua competenza attiva si muoveva tra dialetto e lingua letteraria. Vedremo traccia di ciò nell'analisi linguistica delle lettere. Un impegno a migliorare la propria scrittura emerge, soprattutto negli anni parigini, negli interventi correttori in alcune lettere più formali. ${ }^{10}$

Anche della formazione di Verdi al di fuori di quella musicale non sappiamo molto: ${ }^{11}$ non ammesso, com'è noto, al Conservatorio di Milano, frequentò il ginnasio a Busseto, ma fu in gran parte autodidatta e grandissimo lettore, costruttore in proprio di una cultura molto vasta e aperta sia alla classicità che alla modernità italiana e ancora di più straniera, che emerge con tutta evidenza dalle lettere; non ha lasciato traccia di una formazione linguistica e grammaticale, ma sappiamo che Giuseppina Strepponi, colta e più attenta del marito al rispetto della grammatica, ne revisionò e corresse spesso, possiamo dire dagli anni Cinquanta in poi, le lettere. Ma anche lo stesso Verdi corresse spesso trascuratezze o errori delle minute.

Una prima differenza tra i due epistolari riguarda l'antiletterarietà, molto accentuata e dichiarata in Verdi, mentre Bellini mostra di essere molto più condizionato dalla lingua letteraria e formale. Già la dichiarazione verdiana citata prima sulla pubblicazione delle lettere dei musicisti vi fa riferimento: ma in continuazione nelle sue lettere Verdi fa professione di antiletterarietà nella scrittura epistolare, e soprattutto la dimostra nei fatti. Le lettere di Verdi sono pratiche, comunicative, per lo più immediate, rifuggono dal formalismo, dalla convenzionalità epistolare, dalla retorica, dal lessico letterario e aulico. Sono influenzate dalla lingua colloquiale e informale, con le sue marcatezze, le sue brevità, le sue sconnessioni sintattiche, le sue sgrammaticature, molto più che non dalla lingua letteraria.

A differenza di Verdi, poco rispettoso delle convenzioni testuali epistolari, ${ }^{12}$ Bellini segue la "grammatica espistolare" nelle formule di esordio e congedo, nella struttura del testo, nella prossemica. Usa voci letterarie e burocratiche per innalzare il dettato (postergare, divagamen-

10 Bellini 2017, p. 26 dell'Introduzione.

11 Nell'ambito della sterminata bibliografia verdiana, citerei come particolarmente utili a questo fine Oberdorfer-Conati 2001, Mellace 2013; informazioni utili soprattutto a partire dalle lettere anche in Buroni 2016.

12 Serianni 2002 (ma già 1996), Antonelli 2004. 
to, fallenza 'colpa', ecc.), e, come vedremo, tende spesso a un periodare ampolloso infarcito di figure retoriche. In grammatica, alterna forme moderne o popolari alle forme letterarie e tradizionali, molto presenti (tipo io avea). Anche il lessico melodrammatico, con i sintagmi tipici della librettistica (ciglia piangenti), compare nelle sue lettere, pur se talvolta in tono scherzoso.

Caratteri in parte comuni ai due sono invece la variazione diacronica interna e soprattutto la variazione di registro a seconda dei destinatari.

Per la diacronia interna, va naturalmente sottolineata la ben diversa durata temporale dei due epistolari: quello di Bellini occupa 16 anni, dal 1819 al 1835, mentre quello di Verdi va dal 1829 (ma una sola lettera, poi si passa al 1832) al 1901, per una estensione di ben 72 anni.

Nel caso di Bellini, si nota un'evoluzione verso un maggiore controllo linguistico e grammaticale, e anche la riduzione del regionalismo fonomorfologico, soprattutto dopo il soggiorno a Milano (1827-1833), e dopo il trasferimento a Parigi (1833-). Se nelle prime lettere (1819) il controllo e l'adesione ai modi formulari e al rispetto della correttezza grammaticale, molto vistosi e in qualche modo forzati, sono chiaramente frutto di aiuti esterni, più avanti negli anni aumenta la sua padronanza linguistica e quindi anche il controllo. Pur nell'ambito di una durata circoscritta a 16 anni, sono evidenti la svolta e l'apertura a orizzonti culturali più ampi insieme a una maggiore competenza e cura della lingua italiana. ${ }^{13}$

Nel caso di Verdi, una evoluzione generale nel tempo mostra fondamentalmente una maggiore presenza nei primi anni di lettere molto formali e non del tutto esenti da cadute verso il basso, una certa crescita della padronanza linguistica nel tempo, ma soprattutto un allargamento progressivo negli anni centrali della sua vita verso un variato ventaglio di stili scrittorii in linea con la sua crescita artistica, culturale, professionale e umana; negli ultimi anni si nota un evidente asciugamento nella misura del testo e del periodare.

La variazione diafasica o di registro in relazione ai destinatari è evidentemente un dato comune a tutti gli epistolari di scriventi colti o

13 Sono documentate correzioni linguistiche delle minute. 
di media competenza linguistica, quindi non ci stupisce che la si riscontri anche nel caso di Bellini ${ }^{14}$ e di Verdi.

Alcune tra le prime lettere di Bellini, soprattutto quelle rivolte a destinatari di grado o ruolo sociale elevato, presentano uno stile formale e controllato con pochi errori o deviazioni (soprattutto fonetici) rispetto all'italiano scritto corretto e, appunto, formale. Escludendo la prima lettera del carteggio, al Duca Stefano Notarbartolo, a cui il giovane Bellini chiede un aiuto per mantenersi agli studi a Napoli, per la quale è accertata una mano esterna,${ }^{15}$ possiamo vedere un esempio di questo stile formale e controllato in un'altra lettera-supplica a un personaggio altolocato e potente, il principe d'Aragona Diego Naselli, del settembre $1819:^{16}$

Eccellentissi>mo Sig.e

Vincenzo Bellini di Catania espone all'E V., che trasportato egli per La musica è venuto seriamente in questa Dominante per essergli più facile di apprenderla in tutta La sua estenzione, onde di tal maniera rendersi utile alla patria non meno, che alla Nazione, a cui appartiene.

È istituzione del Collegio S. Sebastiano che per accordarsi ad un giovane la piazza franca, bisogna prima venire ammesso alla scuola Reale, per così potersene coll'andare del tempo deteger La disposizione, i talenti, l'abilità.

Per non alterar questa Legge il Sig.r Duca Noja capo della Commissione, mesi sono volse ammettere l'Esp<onent`e in detta scuola reale; ma siccome quest'ultimo trovasi bastantemente avanzato nelle teorie musicali a segno tale di credersi vantagioso al Collegio; così volendo vieppiù assicurar l'EV. di quanto espone, La priega a farlo esaminare da' Maestri di detto Collegio, e che verificandosene quanto il Bellini asserisce, si gli accordasse quello che di giustizia gli compete, come a dire La piazza franca, giusta di come si è praticato con tanti altri giovani aspiranti a volersi perfezionare nella musica. Indipendentemente a ciò fa riflettere l'Esp $<$ onent`e all'E. V. di meritarsi tale grazia, e per appartenere ad un'antico impiegato, e per l'inabilita-

14 Sulla variazione in due livelli della lingua delle lettere di Bellini cfr. Nicolodi 2004 p. 4 sgg.

15 Pastura 1959, p. 39, Bellini 2017, n. 2.

16 Questa lettera, la n. 4/2 in Bellini 2017, è preceduta da un'altra allo stesso destinatario e con la stessa data, la n. 4/1, che contiene la medesima supplica in una versione in parte diversa nei contenuti, e meno controllata nella lingua. Si dovrà ipotizzare anche qui l'aiuto di una mano esterna. 
zione di un padre onusto, che stentatamente può occorrere al limitato, e semplice mantenimento di una onesta, e numerosa famiglia. mentre sicuro La priega come al Signore

Vincenzo Bellini supp $<$ lican >te

Un caso diverso, ma interessante, di stile formale e corretto è una lettera alla sorella Michela, destinataria, quindi, non superiore socialmente e non 'distante'. Pur dandole del tu, le scrive in un tono davvero formale e quasi sussiegoso, senza deviare da uno scritto-scritto sostanzialmente corretto e neutro:

Mia cara Michela

Questa è la seconda, che ti scrivo, che nell'ultima dello zio D:n Vin:zo vedo che la prima non t'è arrivata, forse per essersi smarrita, pure acchiusa in una del sud:tto zio.

Il nuovo stato dove sei mia cara sorella, mi rende lietissimo, per lo sposo che ha scelto il solo tuo cuore nella persona del tuo Ascanio, pel quale tengo l'idea più intiera d'un giovine pien di talento, amabile $\mathrm{e}$ saggio; tutte queste sue doti per te devono essere un nulla, le/ per lo sprono del tuo amore, che deve sempre pingersi innanzi quanto gli costi al suo cuore, e quai crudi perigli ha dovuto sormontare per possederti: ecco, dunque, unendo la tenera gratitudine alla natura del tuo amore, renderai appieno felice il tuo sposo, che oblierà i suoi passati rancori nel seno del tuo amore e della tua virtù. Esprimi, da mia parte, i più vivi contrasegni d'amicizia al tuo sposo, e presentagli in me un congiunto pien di riconoscenza per l'amore che ti porta. Salutami tutti i tuoi nuovi parenti offrendomi di quanto possano comandarmi in questa; ed infine abb<racciandosti in uno alla tua cara metà, ti prego di ricordarti ne' tuoi contenti qualche volta del tuo fratello

Vincenzo che t'ama ${ }^{17}$

Ma l'epistolario belliniano presenta in massima parte esempi di italiano informale, non ben padroneggiato sul piano grammaticale e sintattico, e interferito dal dialetto sul piano fonetico. Lo mostra soprattutto il ricchissimo contingente delle lettere all'amico Francesco Florimo, suo compagno al Collegio di San Sebastiano, a cui Bellini fu legato da una profonda amicizia e colleganza. A lui il compositore scriveva di tutto, ma naturalmente soprattutto della sua musica, dell'esito delle sue

17 Lettera del 7 luglio 1827, n. 9 in Bellini 2017. 
opere, dei rapporti con librettisti, cantanti, impresari, ecc. Ne portiamo un esempio, tratto dalla lettera da Milano del 2 gennaio 1828, tra i moltissimi che si potrebbero citare:

Mio caro Florimo

[...]

Credea che jeri avessi ricevuto tua lettera con i giornali, come tu nell'ultima mi prometti, e sono restato deluso, e per lettere, e per giornali; perciò credo che sii successo qualche altro impedimento, che cotesto Sig:r Ruffa, nemico dei suoi compaesani ha potuto far nascere: basta: aspetto Vennerdì per vedere che mi dici nella tua che son certo di ricevere: Aspetto pure la decisione di Zingarelli e di te sù la musica che hai veduto, ed a Zingarelli regolati tu come darle la copia intera, perché io quando ti rimetterò il resto dei pezzi te ne manderò un'altra copia intera per te. Sento il denaro che hai pagato a Fabricatore per la Dogana, che credo esser stato troppo, ma quando si ha che fare con negozianti, siamo sempre là; e perciò quello che dovrò spedirti lo farò io, e non mi fiderò più di persona; perché io credea che Ricordi non s'avesse fatto pagare il trasporto; ma tutti sono d'una maniera, tutti Ladri ed ebrei. =

Eccomi a darti notizie de' teatri: L'Elisabetta è restata nell'istesso grado della p<ri>ma sera. Lunedì si fece la pruova generale dell'opera di Donizetti il Borgomastro e questa sera và in scena: io non ho inteso nessuna pruova, ma chi fù alla pruova generale mi disse che nel p<ri>mo atto non v'è niente, che nel 2:do vi è un duetto che forse forse piacerà, nel complesso farà fiasco; ma questa sera si vedrà, ed in ventura ti dirò. $[\ldots]^{18}$

Le lettere di Bellini ai suoi due librettisti, Felice Romani e Carlo Pepoli, sono ricche di interesse in rapporto alla loro collaborazione e all'esplicitazione dei propri principi estetici da parte del compositore. Emerge con chiarezza la grande differenza che Bellini vedeva in Romani e in Pepoli. Con il primo (poeta di 7 delle 10 opere composte da Bellini), aveva una sintonia profonda, a partire da quei principi classicistici che Romani sosteneva con forza sul piano teorico; ${ }^{19}$ nelle lettere a lui il compositore parla più dei cantanti che dei libretti e della musica,

18 La lettera è la n. 12 in Bellini 2017.

19 Su questo aspetto nel rapporto tra Bellini e Romani si ferma Graziella Seminara nella sua introduzione, alle pp. $35 \mathrm{sgg}$.; poco opportuno, in questa sede, un rinvio ad altri contributi su questo nodo dell'opera e in particolare della librettistica della prima parte dell'800, il rapporto tra classicismo e romanticismo. 
senza entrare nel vivo del farsi del testo verbale né del suo rapporto con la musica. Di Romani Bellini apprezzava soprattutto il senso della scena, e la qualità dei suoi versi, valori fondamentali di un libretto. Nella collaborazione con Bellini, Romani, come hanno dimostrato molti studi, ${ }^{20}$ ottiene i migliori risultati di appropriatezza alla musica specie nella versificazione, ma anche negli effetti fonici, con piena sonorità e fluidità, in generale, dei versi. Bellini continuamente nel suo epistolario loda Romani, che chiama "il mio Alfieri", e lo antepone a Pepoli: ma, com'è noto, la loro collaborazione andrò in crisi e si interruppe con Beatrice di Tenda. ${ }^{21}$ Ma restò in entrambi la consapevolezza dell'unicità di quella collaborazione, esplicitata da Bellini in una lettera a Romani del 1834: «con nessun altro poeta che te, non scriverò più opere italiane [...] Scrivi per me solo», ${ }^{22}$ e da Romani nell'orazione funebre per il compositore:

Lottammo uniti contro le viziose abitudini del teatro musicale e ci accingemmo concordi a estirparle a poco a poco, a forza di coraggio, di perseveranza, di amore. ${ }^{23}$

Dopo la rottura con Romani, Bellini si rivolge a un "poeta di bel verso, dotato di facilità", ${ }^{24}$ ma privo di esperienza nella poesia per musica: Carlo Pepoli, a cui affida la scrittura del testo dei Puritani (Parigi, 1835). Bellini, che non trova in Pepoli la stessa perizia drammaturgica e la stessa capacità di rendere gli affetti e la passione di Romani, ha con lui confronti e discussioni continue, testimoniati dalle lettere scritte a Pepoli stesso e all'amico Florimo: opponendo resistenza alla raffinata letterarietà del poeta, lo sollecita ad una maggiore naturalezza, e soprattutto verso una scrittura melodrammatica, con una affermazione che resterà come una delle frasi topiche del compositore:

20 Lippmann 1981 e 1993, Mariano 1990, Roccatagliati 1996; sugli aspetti linguistici della collaborazione, cfr. Gaffuri 2010 in Bonomi-Buroni 2010, e BonomiBuroni 2017.

21 Si veda il fondamentale Roccatagliati 1996.

22 Lettera del 7 ottobre 1834, n. 332 in Bellini 2017.

23 Lippmann 1981, p. 358.

24 Parole di Bellini in una lettera a Florimo dell'11 marzo 1834, n. 272 in Bellini 2017. 
[...] Scolpisci nella tua testa a lettere adamantine: "Il dramma per musica deve far piangere, inorridire, morire cantando". Difetto, il voler condotta eguale in tutti $i$ pezzi; ma necessità che tutti questi siano d'una certa maniera impastati da render la musica intelligibile con la loro chiarezza nell'esprimersi, concisa come frappante. Gli artifizii musicali ammazzano l'effetto delle situazioni, peggio gli artifizii poetici in un dramma per musica: poesia e musica per fare effetto richiedono naturalezza e niente più, chi sorte di questa è perduto, ed alla fine avrà dato alla luce un'opera pesante, stupida, che solo piacerà alla sfera dei pedanti; mai al cuore, poeta che riceve per la prima l'impressione delle passioni: e se il core è commosso, s'avrà sempre ragione, in faccia a tante e tante parole che non potranno provare un $h^{25}$

E scrivendo di lui a Florimo durante la composizione dei Puritani, si lascia andare a giudizi davvero molto critici:

[... Mio caro Florimo lodo il tuo zelo nel consigliarmi di finir presto, e ne comprendo tutte le tue possenti raggioni; ma se tu sapessi che ho sofferto e soffro per fare andare innanzi Pepoli è incredibile: il suo naturale è curioso: mette tutto il suo studio nel gioco di combinazioni poetiche, o per dir meglio in certa maniera di risposte, che mi fà perdere la pazienza (resta fra di noi, io lo credo secco secco d'espressioni che abbiano figure, e sentimenti, perciò stenta lo stentabile non nel fare i versi come egli crede, ma dei versi alla mia maniera, che sono quelli che dipingono le passioni al più vivo). ${ }^{26}$

Dal punto di vista linguistico, le lettere ai due poeti sono piuttosto diverse, perché evidentemente diverso era il rapporto con l'uno e con l'altro. Le lettere a Romani ${ }^{27}$ sono scritte in una lingua piuttosto neutra, controllata e abbastanza corretta, di cui possiamo vedere il seguente esempio:

Mio caro Romani

Volevo scriverti ieri, ma non è stato possibile; sono assediato da visite e da presentazioni. Tutti vogliono conoscermi e congratularsi con me; e sì che sono nella patria di Donizetti!... Quanti ne schiatteranno di rabbia! Allegramente!

25 Lettera a Carlo Pepoli dei primi di giugno 1834, n. 291 in Bellini 2017.

26 Lettera a Florimo del 4-5 agosto 1834, n. 314 in Bellini 2017.

27 In tutto 9 in Bellini 2017. 
La nostra Norma fece deciso furore. Se tu la sentissi qui com'è eseguita, la crederesti quasi cambiata; a me pare un'altra: mi fa un effetto mirabile. Ha sbalordito tutti i Bergamaschi, e quanti forestieri erano in teatro: Bresciani, Veronesi ed anche Milanesi; è un vero trionfo! Tutto è più vivo, i cantanti si sono impadroniti della parte, e vi mettono molta anima. La Giuditta è di buon umore, e in voce e canta e declama in modo da strappare le lagrime... Fa piangere anche me!... E piansi infatti per tante emozioni che provai dentro nell'anima... Ti desideravo a me vicino per dividerle con te, mio buon consigliere e collaboratore, perché tu solo mi comprendi e la mia gloria non va disgiunta dalla tua. La Taccani è una brava Adalgisa, figura bene, e dice come va detta la sua parte, e nel Sola furtiva al tempio riscosse uno scoppio di applausi unanimi. Reina, che non è Donzelli, vi mette tanto fuoco da duplicare la sua voce e la sua persona. Pronunzia chiaro e si muove con energia; è un Pollione innamorato davvero e feroce: tu lo troveresti esagerato per un proconsole romano. Dice tragicamente Meco all'altar di Venere, ma fa piangere ed è molto applaudito nella stretta Me protegge me difende. Il terzetto non può essere eseguito meglio: lo agiscono bene e con forza; fece rabbrividire tutti, e lo trovano un bel finale, anche senza il concerto di pertichini, Druidi, Druidesse e altri cori da far chiasso. Tu avevi ragione ad essere ostinato che fosse così... Ti hanno fatto perdere la pazienza!... Ma ora son contento anch'io. Gli applausi e le chiamate al maestro ed ai cantanti furono assai e spontanei ed universali. Se tu fossi stato qui, ti avrebbero chiamato sul palco, tanto la poesia piace: la trovano tragica e sublime. Ma tu, secondo il solito, non avresti voluto comparire, e se ti volevano trascinare a forza, saresti piuttosto scappato dal teatro. Sempre secondo il solito: ci conosciamo! Il conte Ottavio Tasca, il conte Secco-Suardi, i Colleoni ed altri sono all'entusiasmo pe' tuoi versi, e mi dicono di dirtelo; te lo scriveranno anche loro co' rimproveri che non sei venuto, ec: ec: È un peccato che tu non mi abbi ascoltato, e non sii qui, perché saresti assai festeggiato, ed avresti molte sodisfazioni di gloria, e saresti contento della nostra Norma e del tuo Bellini, che ti deve tanta riconoscenza e ti ricambia i benefici con altrettanto affetto. Da qui a pochi giorni ritorno a Milano ed avrò assai cose da raccontare. Salutami i nostri soliti amici. Dì al Sansi che non mi dimentico la sua commissione. Come sarà contento il buon vecchio de' miei trionfi! Addio, ricevi i miei abb:cci, e credimi per la vita il tuo Bellini P.S. - Il Mayr ti saluta affettuosamente; mi ha abbracciato e baciato. E sono in Bergamo!... ${ }^{28}$

28 Lettera a Felice Romani del 24 agosto 1832, n. 199 in Bellini 2017. 
Bellini si rivolge a Romani prima con il neutro voi, passando quasi subito al confidenziale $t u$, tornando qualche volta al voi quando il rapporto si tende per i continui ritardi del poeta, causa prima e forse unica del loro progressivo allontanamento che porterà alla rottura. Alle lamentele di Bellini per i ritardi si alternano frequenti dichiarazioni di lode per i suoi versi, e di condivisione per i successi e nel segno del comune senso del tragico e del sublime. Dopo la rottura avvenuta con Beatrice di Tenda, Bellini torna sui modi della loro rottura, proponendo una riappacificazione e una rinnovata collaborazione, che certo sarebbe avvenuta se Bellini non fosse prematuramente scomparso. Interessante è questa lettera del 29 maggio 1834 (lett. 286/2), scritta dal compositore durante la composizione dei Puritani, che contiene giudizi severi sul nuovo librettista:

[...] se di tale verità eri convinto dovevi scrivere e publicare su tutti i giornali un'accanito libello come facesti? E la tua coscienza lo comportò? Non fui tutto il Giugno, Luglio, sino al 10: Agosto alla tua porta? Dopo il mese di Agosto sino al 10: Settembre che fui a Bergamo per montare la Norma non mi portai a Milano ove restai fino al 7: Dicembre? E poi, che non aggiungesti d'offese senza necessità e fuori questione? E tu dici di avermi molto amato? Io ti credo benissimo, ma nel mio cuore lagrimava - la Pasta e Papadopoli e l'istesso Cartagenova sono testimoni - quando t'incontrava nelle strade di Venezia in quell'epoca sciagurata che mai oblierò in mia vita; sì, t'incontravo, ${ }^{29} \mathrm{e}$ con sensibile pena mi dicea: Dunque dovrò romperla con chi mi procurò tanta gloria? Che fu l'amico dei più segreti miei pensieri? Ma le tue ultime linee dell'avviso nella Beatrice ed il mio animo allora irritato per tanti diversi dispiaceri che l'opprimevano, non mi rimossero da far stampare lo sciagurato articolo sul giornale di Venezia. Ma tiriamo un velo sù quanto è successo; e se mi ami, pensiamo a riparare con comune decoro al mal fatto e riaccendiamo la nostra affezione appena sopita, che non si spense, a dispetto del nostro grandissimo irritamento, che ha fatto gioire non pochi. Ritorniamo amici meglio che non lo siamo stati, e siam degni l'uno dell'altro! A te non manca talento per scrivere, come articolo, di comune consenso fatto inserire nei giornali: che appresso le cure datesi d'alcuni amici presso il Romani ed il Bellini sono riusciti a riunirli per nuovi lavori; questi ultimi tengono per non proferite tutte

29 Si noti l'alternanza, molto comune all'epoca soprattutto nelle scritture di media formalità, tra il più frequente imperfetto tradizionale in $-a$ e quello più moderno in $-o$, che Manzoni avrebbe di lì a poco preferito nella versione definitiva del suo romanzo. 
le espressioni d'offesa che si trovano negli articoli della questione ec: Ciò che ti propongo è la formula che s'usa in Francia, quando i secondi giungono a pacificare dei duelli; i giornali annunciano la querela finita nel modo che sopra ti dico: così alcun di noi non resterà avvilito: diversamente sarebbe degno dell'altro. Non siam noi per evitare un duello; ma per riabbracciarci ed essere legati in tenera amicizia; quindi consiglia il tuo cuore, come io ho consigliato il mio, e da saggio deciditi. Io sarei volentieri venuto a Milano per tutto accomodare di persona: ma per ora Milano non mi presenta che seri dissapori e m'è forte necessità restarne ancora lontano per mia quiete; e poi mi trovo aver dato parola a $\langle$ Pepoli $>$ per libro che scrive. Questo povero giovane ha molto talento; ma il teatro è assai difficile e io sono ancora più difficile del teatro stesso. Spero di portarlo a fine, ma soffro molto senza i tuoi versi e il tuo talento, e poi, poi, oh, taci a tutti tale sua inespertezza, per non scoraggiarlo più che mai. Mi si invita a scrivere un'opera a Napoli per la primavera prossima; se comporrò verrò a Milano in gennaio ed allora, s'è destino di tornare ancora amici, scriveremo. Addio mio caro Romani, se tu non rispondi sarà l'ultimo che ti dà

Il tuo Bellini

Le lettere al bistrattato Pepoli ${ }^{30}$ sono decisamente più confidenziali (gli dà subito del $t u$ ), espressive, vivaci, e stemperano in un tono amichevole e scherzoso le critiche al suo modo di poetare un libretto, riferite in modo specifico a singoli passi del testo, sui quali Bellini si ferma anche in relazione alla componente drammaturgica, che lamentava carente nel poeta:

Mio caro Carluccio

II terzetto và bene, come l'hai accomodato. Nel fare il Coro che apre la parte 2:a, mi sono avvisto che è assai corto; quindi cerca d'allungarlo sino a otto versi, e cerca di fare un Coro che possa essere degno dei versi che dice Giorgio: pensaci bene, caro carino. Se il libro verrà un eccellente pasticcio, almeno che la poesia 〈non〉 sia comune, ma dettata dal genio Pepolico! Hai capito? ... Ancora una piccola cosa: al coro, Coro, Qual novella? G<iorgi>o Or prende posa. Coro miserella! è insana ognor? Qui invece di attaccare Cinta di Rose ec:, vorrei che Giorgio potesse rispondere $A b$ ! si ognor (p<er〉 esempio), il Coro, senza tregua? Giorgio allora dica Accostatevi, ascoltate, (p<er> esempio) o cosa simile ec: ec: - Così la scena viene preparata, e l'attenzione si presterà con più

30 In tutto 7 in Bellini 2017. 
interesse al cominciamento del racconto. - Quando poi finirai qualche cosa, vieni: ed anche se ti sorgesse qualche difficoltà<,> vieni sempre a vedere il tuo suplizio che ti ama a dispetto della tua testina dura.

Addio

Il tuo Bellini. ${ }^{31}$

Si notino, nel segno dell'espressività e del colloquialismo, contrapposto alla sostanziale letterarietà dello stile delle missive a Romani, gli alterati (carino, testina), il neologismo scherzoso pepolico.

La variazione diafasica è particolarmente evidente nella scrittura epistolare di Verdi. Il tono differente usato da Verdi verso i diversi destinatari è motivato naturalmente da vari fattori: rapporto sociale con il destinatario (età, posizione socio-culturale), rapporto professionale (impresari, librettisti: ma con ciascuno dei suoi librettisti si rapporta in modo molto diverso) e amicale/affettivo. Si evidenzia una spiccata differenziazione dei registri e una diversissima incidenza della componente espressiva, sempre connessa con l'affettività amicale: Verdi, la cui scrittura è in generale asciutta e concreta, concentra l'espressività nelle lettere rivolte a pochi destinatari-amici: lo scultore Vincenzo Luccardi, l'amico Opprandino Arrivabene, il librettista Piave.

Con lo scultore friulano Vincenzo Luccardi, che scriveva in un italiano stentato, quasi da semicolto, Verdi ebbe una fitta e continuativa corrispondenza (dal 1844 al 1876, anno di morte di Luccardi), ${ }^{32}$ informata a cordiale amicizia, intimità scherzosa, forte espressività: corrispondenza in gran parte incentrata sull'attività teatrale romana, di cui Luccardi, che viveva a Roma e ed era 'buon gustaio di musica', come lo definisce Verdi, lo informava; ma i due si scrivevano anche di arte in generale, di politica (Luccardi era clericale e legato al Papato).

Analogie sul piano linguistico e comunicativo si riscontrano nel carteggio con un altro grande amico, il giornalista e critico Opprandino Arrivabene, ${ }^{33}$ uno degli esempi più significativi dei nuovi rapporti e dell'allargamento della cerchia delle amicizie di Verdi con l'ingresso in Parlamento.

Ma il grado di espressività amicale che Verdi usa con Luccardi è

31 Lettera a Carlo Pepoli dell'8 settembre 1834, n. 320 in Bellini 2017.

32 Luccardi 2008.

33 Turba 2018. 
molto simile soprattutto a quello che usa con il librettista con cui collaborò più a lungo, Francesco Maria Piave, con cui Verdi corrispose dal 1843 al 1867, fino alla grave malattia del librettista che lo porterà alla morte. Con Piave, com'è ben noto, Verdi si trovò molto bene: la sua dedizione al Maestro e la sua malleabilità furono elementi nodali nella loro collaborazione e nel complesso Piave si rivela, nonostante l'opinione diffusa e il livello letterario mediocre, librettista molto funzionale per la drammaturgia e la musica verdiane. ${ }^{34} \mathrm{Il}$ loro rapporto fu improntato a un'amicizia e a una confidenza che Verdi non ebbe con nessun altro dei suoi librettisti, e che appaiono tra le più strette di tutto il giro dei suoi corrispondenti. Componente dominante della loro comunicazione fu lo scherzo affettuoso, a cui si lega un lessico particolarmente espressivo e giocoso: Verdi chiama Piave con nomignoli di cui solo in parte cogliamo il significato e l'allusività: gatto (allusione al carattere sornione di Piave), poeta-gatto, ludro (veneziano "uomo grasso a forma di otre'), e molti altri. E nello scherzo affettuoso rientra tra l'altro una particolarità allocutiva che Verdi usa solo con Piave, alternando il consueto $t u$ a un lei scherzoso:

[...] farai da Gallo mettere il cembalo buono assai. Ha capito sig. Ludro? Sperava da te un'altra lettera sulla 2 recita del Lorenzino ma Lei è un ludro. Intanto mi rallegro con te e col teatro di quest'esito. ${ }^{35}$

Molto diversi i registri e le modalità pragmatiche che Verdi segue con gli altri librettisti, condizionati dal grado di confidenza e dal rapporto di età, dalla considerazione per la loro qualità letteraria e drammaturgica, dalla maggiore o minore difficoltà di collaborazione nella composizione delle opere e dei libretti, e così via. Non sempre l'uso del pronome allocutivo più confidenziale, il $t u$, è segno di amicizia e di facilità di rapporto: Verdi lo usa con Solera, ${ }^{36}$ che stimava, ma con il quale il rapporto presto si incrinò, con Piave appunto, con Maffei, di cui come si sa fu molto amico. Il rapporto con Somma, quasi coetaneo del Maestro, non fu mai molto caldo, improntato a correttezza e rispettosa

34 Per una caratterizzazione complessiva della collaborazione tra Verdi e Piave si veda d'Angelo 2017.

35 Lettera a Francesco Maria Piave del 9 marzo 1845, in Verdi 2012 n. 58.

36 Molto scarsa la documentazione di lettere fra i due: Cesari-Luzio 1913 a p. 440 riportano un'unica lettera di Verdi a Solera, del 25 dicembre 1845. 
distanza: Verdi, che gli si rivolge sempre con il non marcato voi, usa con lui in alcuni casi 'maniere un po' rudi', che riflettono la scarsa sintonia sul piano artistico. ${ }^{37}$

Un tono costante di relativa distanza, non di confidenza, caratterizza le lettere a Salvadore Cammarano, poeta per il quale Verdi professa e dichiara sempre grande ammirazione: Verdi gli si rivolge prima con il più formale lei, poi con il neutro voi. I rapporti si fecero più difficili durante la travagliata composizione del Trovatore.

Il rapporto con Ghislanzoni, ${ }^{38}$ unico dei librettisti a cui Verdi si rivolge con il lei, è decisamente più freddo, asciutto e soltanto pragmatico rispetto a quello, pur variegato, con gli altri librettisti. Le lettere ad Antonio Ghislanzoni si possono dividere tra un nutrito gruppo concentrato nel 1870, anno di composizione di Aida: sono improntate tutte a uno stile asciutto e molto pragmatico, del tutto privo dei convenevoli e delle espressioni amichevoli che Verdi riserva ad altri destinatari. Le divergenze sulla composizione del libretto sono molto rilevanti, e Verdi assume quasi sempre un tono critico. L'asciuttezza e la evidente velocità di scrittura di queste lettere comportano caratteri linguistici molto spiccati: estrema brevità sintattica; trascuratezze morfologiche, specie nei pronomi; costrutti oralizzanti di sintassi marcata e anacoluti:

Ricevei jeri il Finale, oggi il Duetto che và bene salvo il Recitativo che secondo me, mi scusi, si poteva dire ancora con minori parole; ma ripeto può stare bene così. [...]

Parmi che le Litanie, e scusi per la millesima volta l'ardire, dovrebbero essere di strofette di un verso lungo ed un Quinario. Oppure, e sarebbe forse meglio per poter dir tutto, di due versi ottonarj ed il quinario che sarebbe l'ora pro nobis. (22-8-1870)

Le ripeto per la ventesima volta: Io non desidero che una cosa: riuscire. (24-10-1870)

Le lettere successive al 1870, conclusa la composizione di Aida,

37 Verdi gli rimproverava mancanza di brevità e incisività, incapacità di scolpire in poche parole l'immagine su cui agisce l'efficacia drammatica e musicale del compositore, quel concetto che più tardi il compositore riassumerà nel sintagma 'parola scenica'.

38 Il carteggio tra i due è in corso di preparazione per l'Edizione Nazionale, a cura di chi scrive, Edoardo Buroni e Marco Spada. 
sono molto più amichevoli rispetto alle precedenti, e Verdi passa al $t u$.

Con Boito $^{39}$ Verdi ebbe, com'è noto, un rapporto complesso che evolve nel tempo, partendo da una contrapposizione, anche se non riflessa nelle lettere (Boito wagneriano, di lui traduce alcuni libretti) per arrivare a una profonda sintonia e intesa. Ancora durante la composizione di Otello si nota un certo distacco, che poi per Falstaff lascia il posto ad una intesa profonda, a una grande confidenza, nonostante la differenza di età, nel segno di una parità artistica.

Un ultimo accenno ${ }^{40}$ alle lettere al suocero Antonio Barezzi, si può dire l'unico parente con cui Verdi ebbe scambio epistolare. ${ }^{41}$

Nelle non numerose lettere a Barezzi, non edite in carteggio indipendente, e non studiate, emergono tratti caratteriali e scrittorii interessanti. Verdi gli si rivolge con tono formale, ma diretto e franco, del tutto privo di cerimoniale, naturalmente con il lei, che manterrà sempre. Il tono di rispetto ma anche di affetto sincero e profondo che informa le sue lettere al 'benefattore', come lo chiama spesso, è proprio soltanto di questo rapporto ed è alla base di una lingua neutra, mai vivace ed espressiva, ma piena di scorrettezze linguistiche, specie nel delicato settore pronominale. Emerge in particolare in queste lettere una interessante documentazione della varietà di forme per gli allocutivi di cortesia. Prendendo ad esempio l'importante lettera da Parigi del 21 gennaio 1852, in cui Verdi entra tra l'altro nel delicato argomento del suo legame con la Strepponi, troviamo, per fare solo un esempio, per il pronome di cortesia in forma tonica, in funzione di soggetto, Lei, Egli, ei, Ella stessa:

Non credo che per propria ispirazione m'avrebbe scritto una lettera che Egli sa non poteva che farmi dispiacere: $\mathrm{ma}$ ei vive in un paese [...]. Sia giudice Ella stessa, e sia giudice severo, ma freddo e spassionato. ${ }^{42}$

Dopo questa breve carrellata sulla variazione linguistica in rap-

39 Boito 2014.

40 Escludo in questa sede riferimenti all'importante carteggio con Clarina Maffei (su cui Cella 2014), in preparazione per l'Edizione Nazionale.

41 Qualche lettera, negli ultimi anni, Verdi scrisse alla figlia adottiva Maria Verdi sposata Carrara.

42 In Verdi 2012, n. 152. 
porto ai destinatari, mi fermo ora brevemente su alcuni aspetti linguistici generali, con taglio comparativo fra i due carteggi.

Il regionalismo è molto più presente nella scrittura epistolare di Bellini ${ }^{43}$ che in quella di Verdi.

Bellini parlava soprattutto dialetto, come quasi tutti all'epoca, almeno quando si trovava nella sua città, e forse anche a Napoli con i condiscepoli: al di fuori di Catania e Napoli, nei soggiorni a Milano, Venezia, per non parlare di Parigi, Bellini avrà parlato presumibilmente quell'italiano stentato, misto di dialetto e di lingua letteraria, che le persone di cultura usavano all'epoca come lingua di comunicazione.

Nella sua scrittura epistolare entrano elementi dialettali soprattutto fonetici (incerto uso delle consonanti doppie in forme come colleggio, pegiore, ribbattere; sonorizzazione delle occlusive postnasali come anzioso, convingersi; assordimento di consonanti sonore come compinare), morfologici e sintattici (p.es. avere per essere, congiuntivo pro condizionale: Parigi, paese che mi piacesse più di Londra; l'uso della preposizione a premessa al complemento oggetto: senti a Bellini). Molti di questi fenomeni dialettali scompaiono o diminuiscono dopo il 1830. La presenza dialettale nel lessico emerge in numerosi meridionalismi, tra i quali p.es. capire 'contenere', partimento, sparambiare 'risparmiare'.

In Verdi la componente dialettale e regionale è decisamente più contenuta. Poco presente nella grafia e nella fonetica, emerge in qualche forma verbale come il condizionale in -essimo del tipo avressimo, abbastanza frequente, e in alcune voci dialettali, prevalentemente, ma non solo, settentrionali, usate con finalità denotativa o connotativa: magut 'muratore', spegasc 'brogliaccio'; rari inserti milanesi in mescolanza di codice (nun sem nun... Milan...el prim teater del mond!).4 Qualche venetismo nello scherzoso scambio con Piave, come visto sopra.

Il lessico nel suo complesso appare piuttosto diverso tra i due compositori, anche se prevedibilmente alcune categorie di voci sono comuni, ma presenti in misura diversa.

Le voci espressive, tipiche dell'oralità, come alterati, interiezioni, epiteti affettivi o, di più, ingiuriosi (asino, bestione, cane rivolti ai can-

43 Cfr. Nicolodi 2004, p. 4 sgg.

44 Lettera a Giulio Ricordi del 5 novembre 1877, n. 459 in Verdi 2012. 
tanti) o, fino ai trivialismi (fottere, coglionare), sono più frequenti in Bellini, che mostra anche un buon grado di creatività nei neologismi effimeri, come antegalantuomo 'mascalzone', pepolico riferito a Pepoli. Espressività e colloquialismo colorito sono riferiti in gran parte alla vita teatrale.

Se questa categoria di voci è senz'altro più abbondante in Bellini che in Verdi, il secondo presenta una maggiore apertura verso le parole straniere. Non si contano in Verdi i francesismi, usati a piene mani in particolare in lettere in cui si parla di cose francesi e di Parigi, ma anche per i campi semantici dello spettacolo e della vita in generale (politesse, verve, nonchalant, e così via); non mancano casi di passaggio sciolto dall'italiano al francese. Non occorre ricordare la totale padronanza che Verdi aveva della lingua francese, molto superiore a quella di Bellini. Ma oltre ai francesisimi, Verdi usa anche qualche anglicismo: meatings, budget, spleen, Luna di miele, strada di ferro, ecc. Naturalmente anche nelle lettere di Bellini, soprattutto da Parigi ma anche prima, a partire dal soggiorno milanese, ricorrono numerosi francesismi, sia relativi alla quotidianità (argent, armoir, routine), sia ovviamente, e soprattutto, alla vita teatrale (balcone 'galleria', pièce anche nel senso di 'libretto', mise en scène, ecc. $)^{45}$.

Alla differenza nell'uso delle voci letterarie, presenti in Bellini e assenti in Verdi, ho già fatto cenno.

Una categoria del lessico molto importante, sia sotto il profilo linguistico, sia sotto il profilo musicologico, è, oltre a quella delle voci dell'opera, scontate negli epistolari dei compositori, quella dei termini e delle espressioni che sono spia della concezione estetica dell'autore. Qualche esempio per Bellini ${ }^{46}$ attore-cantante, situazione, stretta, canto spianato (cioè 'sillabico', in cui a ogni nota corrisponde una sillaba), canto vibrato; e per Verdi: selva/tela 'struttura iniziale del dramma, stesa dal compositore', effetto, posizione, finitezza di canto.

Un aspetto in cui la scrittura epistolare dei due musicisti diverge nettamente è la sintassi, spia da un lato della diversa padronanza linguistica, dall'altro delle differenze di temperamento e di personalità.

45 Cfr. Nicolodi 2004, pp. 14-16.

46 Questa categoria di voci è molto ben analizzata da Graziella Seminara nella sua Introduzione ai Carteggi belliniani (pp. 26-33); si vedano anche Beghelli 2004 e Nicolodi 2004. 
La sintassi di Verdi è quasi sempre breve, asciutta, incisiva e pragmatica, spesso perentoria, e sempre di più nel corso degli anni:

[...] E Noi?...Io avrei amato una politica più generosa, e che si pagasse un debito di riconoscenza. Centomila dei nostri potevano forse salvare la Francia e Noi. In ogni modo avrei preferito segnare una pace vinti coi Francesi, a questa inerzia che ci farà disprezzare un giorno. La guerra Europea non l'eviteremo, e noi saremo divorati. Non sarà domani ma sarà. Un pretesto è subito trovato. Forse Roma... il Mediterraneo... E poi non vi è l'Adriatico che Essi han già proclamato Mare Germanico? $^{47}$

E si noti in questo passo un costrutto oralizzante come la dislocazione a sinistra la guerra Europea non l'eviteremo.

La sintassi di Bellini è molto più variata sul piano diafasico e diamesico, spesso ridondante e prolissa, tendente da un lato alla letterarietà e alla retorica nelle lettere più formali, e dall'altro piena di costrutti del parlato, trascuratezze, sconnessioni. Un periodare lungo, paratattico, quasi flusso di un dettato interiore ricorre soprattutto nelle lettere più spontanee e con grado di affettività alto rispetto al destinatario.

Spesso poi emerge la tendenza a un periodare ampolloso ma faticoso, come di chi cerca di elevare la scrittura ma non la padroneggia bene, come nell'esempio che segue:

sebbene non ho il piacere di conoscerli da vicino, pure non gli saranno discari le offerte di mia riconoscenza per tutto quello che hanno fatto eseguire in mio vantaggio, ed in onore del nome Siciliano, che sebbene lontano da più anni, pure le contrade della mia patria mi sono sì care che i due primi soggetti che sin'ora ho posto in musica sono nel suolo della Sicilia accaduti: il primo Carlo Duca d'Agrigento, in S. Carlo, il secondo, Il Pirata, alla Scala: tutto ciò ho il piacere che il Cavaliere glie lo dica, perché eglino amano la loro/ patria e non poco; e di ciò ne è pruova il felice governo che ebbe la Sicilia negli anni che il prin<cispe Campofranco ne fù Vice-Rè; infine li preghi d'annoverarmi nel numero dei loro più grati amici, e abbino di certo, che cotesto loro tratto, mi sarà scolpito per sempre nel mio cuore. ${ }^{48}$

47 Lettera a Clara Maffei del 9 ottobre 1870, n. 374 in Verdi 2012.

48 Lettera a Francesco Florimo del 2 gennaio 1828, n. 12 in Bellini 2017. 
Comune ad entrambi i compositori è, invece, l'abbondanza di frasi nominali, prive di verbo in funzione predicativa, un costrutto che diventerà uno stilema tipico del giornalismo nel secolo successivo, ancora oggi vitalissimo, anticipato dalle recensioni degli spettacoli sui periodici del primo Ottocento. ${ }^{49}$ Qualche esempio, in Bellini:

[...] La Sortita di Rubini un furor tale che non si può esprimere, ed io mi sono alzato ben 10: volte per ringraziare il publico: La cavatina della $\backslash$ prima/ donna pure applaudita: dopo un coro di Pirati con l'Eco, il quale ha fatto un piacer tale, per la novità d'aver immaginato l'eco così bene, $\mathrm{e}$ poi infine entrando dentro le scene cantano sempre per altre 30: battute, e diminuendo sempre le voci con \un'altra/ Horchestra [...]

$\mathrm{E}$ in Verdi, ma in contesto diverso dallo spettacolo, riferito alla sua amata campagna:

[...] Le nostre disgrazie non sono così forti come sul Mantovano e Ferrarese, ma in ogni modo i raccolti tutti sono quasi perduti. Gelsi perduti. Fieni quasi perduti: Frumento una semente o due al più. Melica nasce male e marcisce. Uva quasi niente. $[\ldots]^{50}$

Dall'analisi sono emersi, dunque, aspetti comuni ai due compositori, legati alla loro comune professione e alle analogie in quanto scriventi, possiamo dire semplificando, di media cultura, in un contesto linguistico regionale, ma si sono evidenziate anche molte differenze, in buona parte legate alle loro personalità. La cerchia molto più ampia $\mathrm{e}$ variegata dei corrispondenti verdiani e la ben più lunga estensione cronologica del suo epistolario ne determinano poi una maggiore variazione interna, anche sul piano linguistico.

49 Le recensioni teatrali ma soprattutto di opera sui periodici letterari e culturali, sui quotidiani, sulle riviste di varietà e per le donne, erano improntate spesso nell'Ottocento a uno stile vivace e brillante, molto moderno: cfr. De Stefanis Ciccone 1983 e Bonomi 2017.

50 Lettera a Giuseppe Piroli dell'11 giugno 1879, n. 479 in Verdi 2012. 


\section{RIFERIMENTI BIBLIOGRAFICI}

Antonelli 2004, G. Antonelli, Tipologia linguistica del genere epistolare nel primo Ottocento, Roma, Ed. dell'Ateneo, 2004.

Beghelli 2004, M. Beghelli, Il lessico melodrammatico di Bellini, in Vincenzo Bellini nel secondo centenario della nascita, a c. di G. Seminara e A. Tedesco, Firenze, Olschki, 2004, 2 voll., vol. I, pp. 27-37.

Bellini 2017, Vincenzo Bellini. Carteggi, Edizione critica a cura di G. Seminara, Firenze, Olschki, 2017.

Boito 2014, Carteggio Verdi-Boito, a c. di M. Conati, Parma, Istituto Nazionale di Studi Verdiani, 2014.

Bonomi 2017, I. Bonomi, Note sul lessico musicale nei periodici milanesi della prima metà dell'Ottocento, in Italiani di Milano. Studi in onore di Silvia Morgana, Milano, Ledizioni, 2017, pp. 329-342.

Bonomi 2020, I. Bonomi, Tu, egli, ei, lei, ella, voi...: varietà pronominale nelle modalità pragmatiche della comunicazione epistolare verdiana, in Atti del XII Convegno ASLI, Catania, settembre 2018.

Bonomi-Buroni 2010, I. Bonomi- E. Buroni, Il magnifico parassita, Milano, Franco Angeli, 2010.

Bonomi-Buroni 2017, I. Bonomi- E. Buroni, La lingua dell'opera lirica, Bologna, Il Mulino, 2017.

Buroni 2016, E. Buroni, Lettere e letterature. Una ricognizione del canone e dell'estetica verdiani a partire dai carteggi editi, in Verdi e le letterature europee, a cura di G. Pestelli, Quaderni dell'Accademia delle Scienze di Torino, pp. 29-51.

Cammarano 2001, Carteggio Verdi-Cammarano, a c. di C.M. Mossa, Parma, Istituto Nazionale di Studi Verdiani, 2001.

Cella 2014, F. Cella, Verdi e il salotto milanese di Clara Maffei, in Un duplice anniversario: Giuseppe Verdi e Richard Wagner, a c. di I. Bonomi, F. Cella, L. Martini, Milano, Istituto Lombardo Accademia di Scienze e Lettere, 2014, pp. 165-174.

Cesari-Luzio 1913, I copialettere di Giuseppe Verdi, pubblicati e illustrati da Gaetano Cesari e Alessandro Luzio, Milano, Tip. Stucchi Ceretti \& C., 1913.

d'Angelo 2017, E. d'Angelo, «Invita Minerva». Francesco Maria Piave librettista con Verdi, Foggia, Grenzi, 2017.

De Stefanis Ciccone 1983, S. De Stefanis Ciccone, Saggio introduttivo, in S. De Stefanis Ciccone-I. Bonomi-A. Masini, La stampa periodica milanese della prima metà dell'Ottocento. Testi e concordanze, 5 voll., Pisa, Giardini, 1983.

Gaffuri 2010, V. M. Gaffuri, Felice Romani librettista per Bellini, in Bonomi-Buroni 2010, pp. 75-114.

Lippmann 1981, F. Lippmann, I libretti delle opere di Bellini e lo sviluppo del libretto dell'Opera Seria italiana nei primi decenni del XIX secolo, in M. R. Adamo e F. Lippmann, Vincenzo Bellini, Torino, ERI, pp. 317-362.

Lippmann 1993, F. Lippmann, Felice Romani e Vincenzo Bellini, in Omaggio a Folena, Padova, Editoriale Programma, 1993, vol. II, pp. 1641-1654. 
Luccardi 2008, Carteggio Verdi-Luccardi, a c. di L. Genesio, Parma, Istituto Nazionale di Studi Verdiani, 2008.

Macinante 1995, U. Macinante L'epistolario di Verdi. Un'analisi linguistica, Firenze, Passigli 1995.

Mariano 1990, E. Mariano, Felice Romani e il melodramma, in Opera E libretto, I, Firenze, Olschki, pp. 165-209.

Mattioli 2018, A. Mattioli, Meno grigi più verdi. Come un genio ha spiegato l'Italia agli italiani, Milano, Garzanti, 2018.

Mellace 2013, R. Mellace, Con moltissima passione. Ritratto di Giuseppe Verdi, Roma, Carocci, 2017.

Mengaldo 2003, P.V. Mengaldo, Sullo stile dell'epistolario di Verdi, in Verdi 2001, Atti del Convegno internazionale "La drammaturgia verdiana e le letterature europee", a c. di F. Della Seta, R. Montemorra Marvin e altri, Firenze, Olschki, 2003, pp. $25-45$.

Nicolodi 2004, F. Nicolodi, Appunti sull'epistolario di Bellini, in Vincenzo Bellini cit., vol. I, pp. 1-25.

Oberdorfer-Conati 2001, A. Oberdorfer e M. Conati, Autobiografia dalle lettere, Milano, Rizzoli, 2001.

Pastura 1959, F. Pastura, Bellini secondo la storia, Parma, Guanda, 1959.

Roccatagliati 1996, A. Roccatagliati, Felice Romani librettista, Lucca, LIM, 1996.

Serianni 2002 (ma già 1996), L. Serianni, Spigolature linguistiche dal Carteggio VerdiRicordi (1880-81), in Viaggiatori musicisti poeti, Milano, Garzanti, pp. 162-179.

Somma 2003, Carteggio Verdi-Somma, a c. di S. Ricciardi, Parma, Istituto Nazionale di Studi Verdiani, 2003.

Turba 2018, Lettere di Giuseppe Verdi a Opprandino Arrivabene, a c. di Alessandro Turba, Lucca, Libreria Musicale Italiana, 2018.

Verdi 2012, G. Verdi, Lettere, a c. di E. Rescigno, Torino, Einaudi, 2012. 
